\title{
nota relativa a la determinación de «longitudes de pandeo» en los pórticos metálicos a dos aguas
}

Jesús Ortiz Herrera, Dr. Ingeniero de Caminos 433-2

\section{sinopsis}

En la construcción de naves industriales, frente a la solución de cercha isostáticamente apoyada sobre soportes, se utiliza cada vez más el pórtico metálico a dos aguas de luces pequehas y medias.

Para el estudio de la inestablildad en el plano del pórtico se recurre al método de las ulongitudes de pandeo" que permite una comprobación pleza por pieza, procedimiento discutible, pero generalmente aceptado por su operatividad.

Aunque existen técnicas de análisis numórico que permiten abordar con precisión el problema de la inestabilidad, no 80 pretende con esta Nota introducir innovaciones on ese trata. miento del problema, sino que, aceptando la validez aproximada del mótodo de las longltudes de pandeos, se proponen unas reglas de apllcación inmediata y compatibles con el planteamiento dado por la normativa espanola.

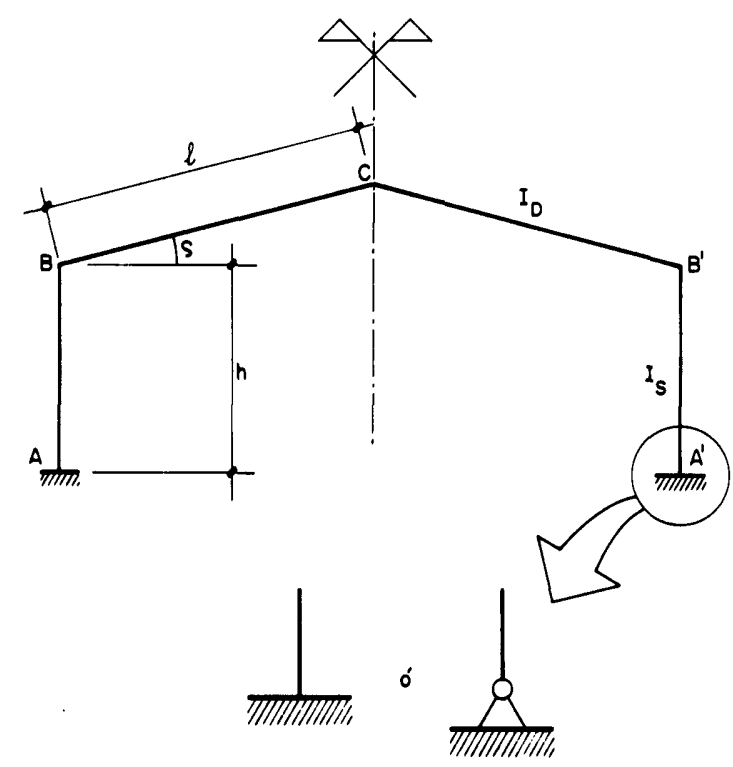

Fig. 1. Pórtico a dos aguas, simétrico, biarticulado o biempotrado.

\section{introducción}

La utilización del pórtico metálico en naves industriales a dos aguas, de luces pequeñas y medias, afianza día a día su competitividad frente a la solución tradicional de cercha isostáticamente apoyada sobre soportes, por diversas razones:

- reducción de costes de fabricación y montaje, susceptibles de compensar el incremento de la cuantía de acero derivado del trabajo de flexión de las piezas;

- adecuación a pendientes reducidas de la cubierta, sin las complicaciones que frecuentemente las mismas provocan en la realización de los nudos de las cerchas;

- posible mejora, en el plano del pórtico, de la longitud de pandeo de los soportes y de las flexiones debidas al viento, lo cual puede compensar parcialmente el encarecimiento de los mismos originado por la introducción de flexiones bajo las acciones verticales de la cubierta. 
No se trata de una solución exenta de inconvenientes: actuación de fuerzas horizontales y momentos sobre las cimentaciones (los segundos pueden eliminarse adoptando pórticos articulados en sus bases); acusada deformabilidad bajo las acciones verticales, que puede limitar el empleo de esta solución al caso de luces reducidas, o imponer variantes de mayor coste unitario (por ejemplo, pórtico con dinteles alveolados). En cualquier caso, no obstante, la posible utilización de la solución pórtico debe ser considerada en este tipo de naves.

Tratándose en general de construcciones modestas, el proyectista generalmente recurre al empleo de métodos de cálculo sencillos, frecuentemente con el único apoyo de tabulaciones y formularios; en este sentido, la comprobación de la inestabilidad en el plano del pórtico (en dirección ortogonal se supone coartada por arriostramientos apropiados) suele efectuarse por el procedimiento de las «longitudes de pandeo», el cual permite reducir el problema real de inestabilidad (pandeo de conjunto) a una comprobación pieza por pieza, método discutible, si bien generalmente aceptado por su operatividad. Pero, en opinión del autor, las longitudes de pandeo de las piezas de este tipo de estructura (fig. 1) no vienen adecuadamente formuladas en la normativa española (refs. 1,2) ni en la internacional (refs. 3, 4, 5, etc.), ni tampoco en obras "clásicas» sobre teoría de pandeo de las estructuras (refs. $6,7,8$ ) o sobre construcción metálica en general (refs. $9,10,11,12$ ).

Por supuesto el problema teórico de la inestabilidad de este tipo de estructuras puede abordarse con precisión por medio de técnicas de análisis numérico establecidas ya desde antiguo (referencias $13,14,15,16,17,18,19$, entre muchas otras). El propósito de esta Nota técnica no es el de introducir innovaciones en el tratamiento teórico del problema, sino, aceptando la validez aproximada del método de las "longitudes de pandeo» (en relación con las posiciones actuales sobre este tema, véase por ejemplo la ref. 20), proporcionar al calculista unas reglas de aplicación inmediata y compatibles con el planteamiento actual del cálculo de las estructuras metálicas según la normativa española.
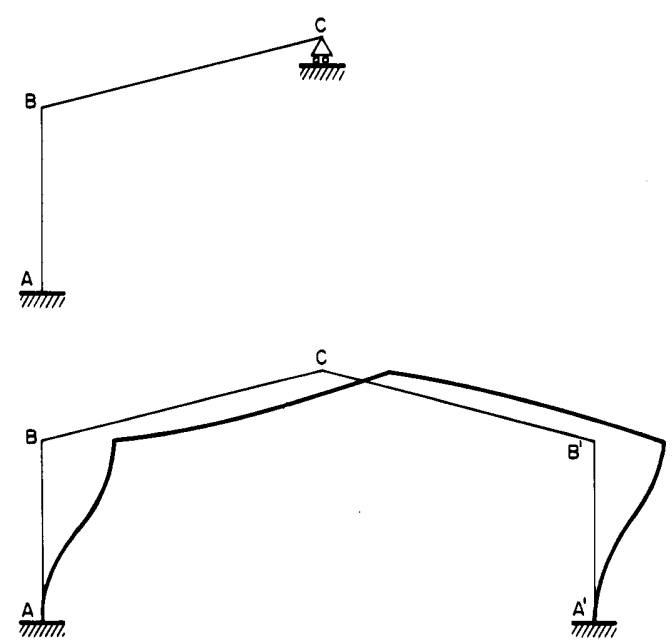

Fig. 2 Modo de pandeo antimétrico.
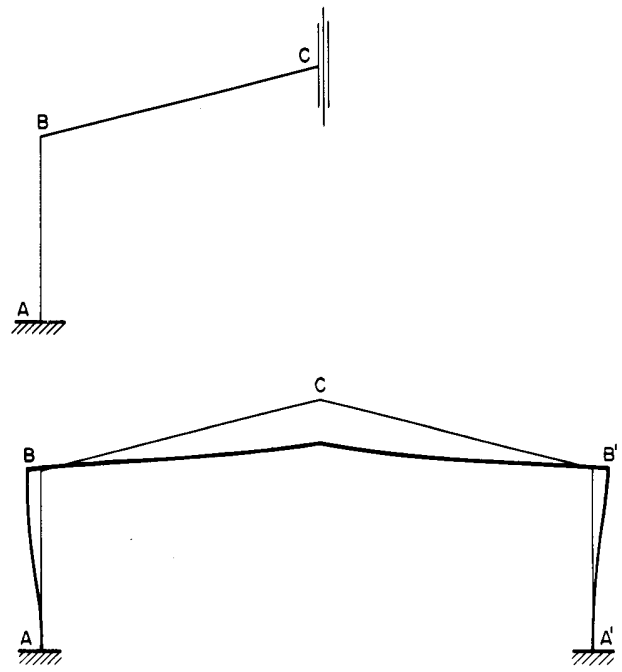

Fig. 3 Modo de pandeo simétrico.

\section{longitud de pandeo de los soportes}

Cada soporte puede contemplarse como una pieza empotrada o articulada en su base, y sustentada elásticamente contra el desplazamiento horizontal y el giro en su extremo superior. En el modo de pandeo antimétrico (fig. 2), el dintel BC introduce simplemente en el extremo superior del soporte una coacción elástica al giro del nudo, de rigidez $3 \mathrm{E}_{D} / \ell$; al ser este valor independiente del ángulo $\varrho$, es claro que el modo de pandeo que se comenta es asimilable al modo de pandeo antimétrico del semipórtico $A B C$ (fig. 2 superior) en el cual el nudo $C$ se abate 
sobre el plano horizontal; es decir, la longitud de pandeo de los soportes será la misma que en un pórtico ortogonal con idénticas características resistentes, y con longitud del dintel igual a 21. Dicha longitud de pandeo puede calcularse, por ejemplo, aplicando los criterios del artículo 3.4.4. (Tabla 3.4.) de la ref. 1, con los grados de empotramiento siguientes:

[1] $K_{1}=1$ o cero (base empotrada o articulada, respectivamente).

[2] $\mathrm{K}_{2}=\frac{\mathrm{I}_{1,} / 2 \ell}{\mathrm{I}_{\mathrm{s}} / \mathrm{h}+\mathrm{I}_{D} / 2 \ell}$

Obtenido el correspondiente coeficiente $\beta$, la longitud de pandeo del soporte en el plano del pórtico es $\beta h$, variable entre $h$ y $2 h$ en pórticos biempotrados, o entre $2 h$ e infinito en el caso de pórticos biarticulados.

Para la aplicación de la expresión [2], debe tenerse en cuenta la posible reducción de la rigidez efectiva del dintel, debido a la correspondiente solicitación de compresión, según se expone en otro apartado posterior.

El modo de pandeo simétrico (fig. 3) es más favorable para la estabilidad de los soportes, ya que, además de una coacción al giro más intensa, el dintel $B C B^{\prime}$ se deforma en dirección horizontal, introduciendo fuerzas de recuperación del desplazamiento del extremo superior de los soportes.

\section{longitud de pandeo del semidintel (BC)}

En el modo antimétrico (fig. 2) no existe, en primera aproximación, desplazamiento relativo de los dos extremos del semidintel BC, y el soporte proporciona un empotramiento elástico en el extremo B del semidintel, pudiendo éste contemplarse como una pieza biarticulada con una coacción elástica al giro en uno de sus extremos, siendo por lo tanto su longitud de pandeo inferior a su longitud real ( $\ell$ ). En cambio, en el modo de pandeo simétrico (fig. 3 ), según se deduce a continuación, se infieren longitudes de pandeo siempre superiores a $\ell$, por lo cual se pasa directamente a considerar dicho modo de pandeo.

Obsérvese la incoherencia de estudiar el soporte y el dintel con diferentes modos de pandeo del conjunto. Evidentemente esto puede considerarse como una simplificación conservadora, ya que cada barra se analiza bajo las vinculaciones que le son más desfavorables. No obstante, es claro que el modo de pandeo (simétrico o antimétrico) será uno determinado para cada estructura particular.

Para el estudio del pandeo del dintel se considera la subestructura representada en la figura 4, en la cual la única solicitación tenida en cuenta es el esfuerzo axil, $N^{*}(>0$; compresión), del dintel. De esta forma, habitual en la determinación de longitudes de pandeo, se prescinde de las flexiones «primarias», transformando el problema de pandeo gradual («divergencia» de equilibrio) en un problema de bifurcación de equilibrio; conocida la longitud de pandeo, la comprobación de la pieza solicitada en flexión compuesta se efectúa mediante criterios sobradamente conocidos.

En el extremo 1 del semidintel (fig. 4) se han representado las coacciones elásticas al giro (C) y al desplazamiento horizontal ( $C^{\prime}$; en realidad, acopladas) introducidas por el soporte; de modo conservador, se considera sólo la coacción C, lo cual subvalora la vinculación de la pieza y, 
por consiguiente, incrementa su longitud de pandeo. Por conveniencia formal se define el parámetro adimensional siguiente (relación de rigideces):

$$
\begin{gathered}
|3| \mu=C l /\left.4 E\right|_{1, ;}-\frac{4 E I_{s}}{h} \text { (soporte empotrado) } 0 \\
\frac{3 E L_{s}}{h} \text { (articulado) }
\end{gathered}
$$

Aceptando que la pérdida de la forma recta se produce de modo inextensible, los corrimientos $u$ y $v$ (fig. 4) están relacionados entre si: $v=u$ ctg $Q$, quedando reducidos los grados de libertad de la barra a dos $\left(u, \omega_{1}\right)$. Denominando

$$
\mathbf{u}^{\prime}=\left[\frac{\mathrm{u} \operatorname{sen} \varrho}{\ell}, \omega_{1}\right]
$$

los movimientos extremos de la barra se expresan:

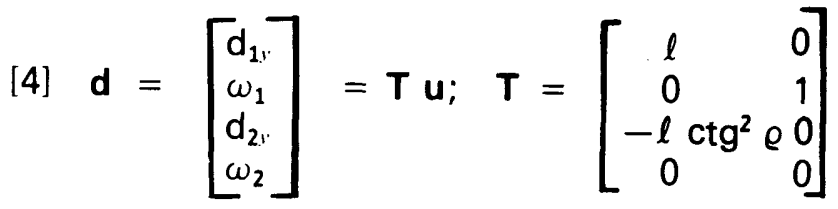
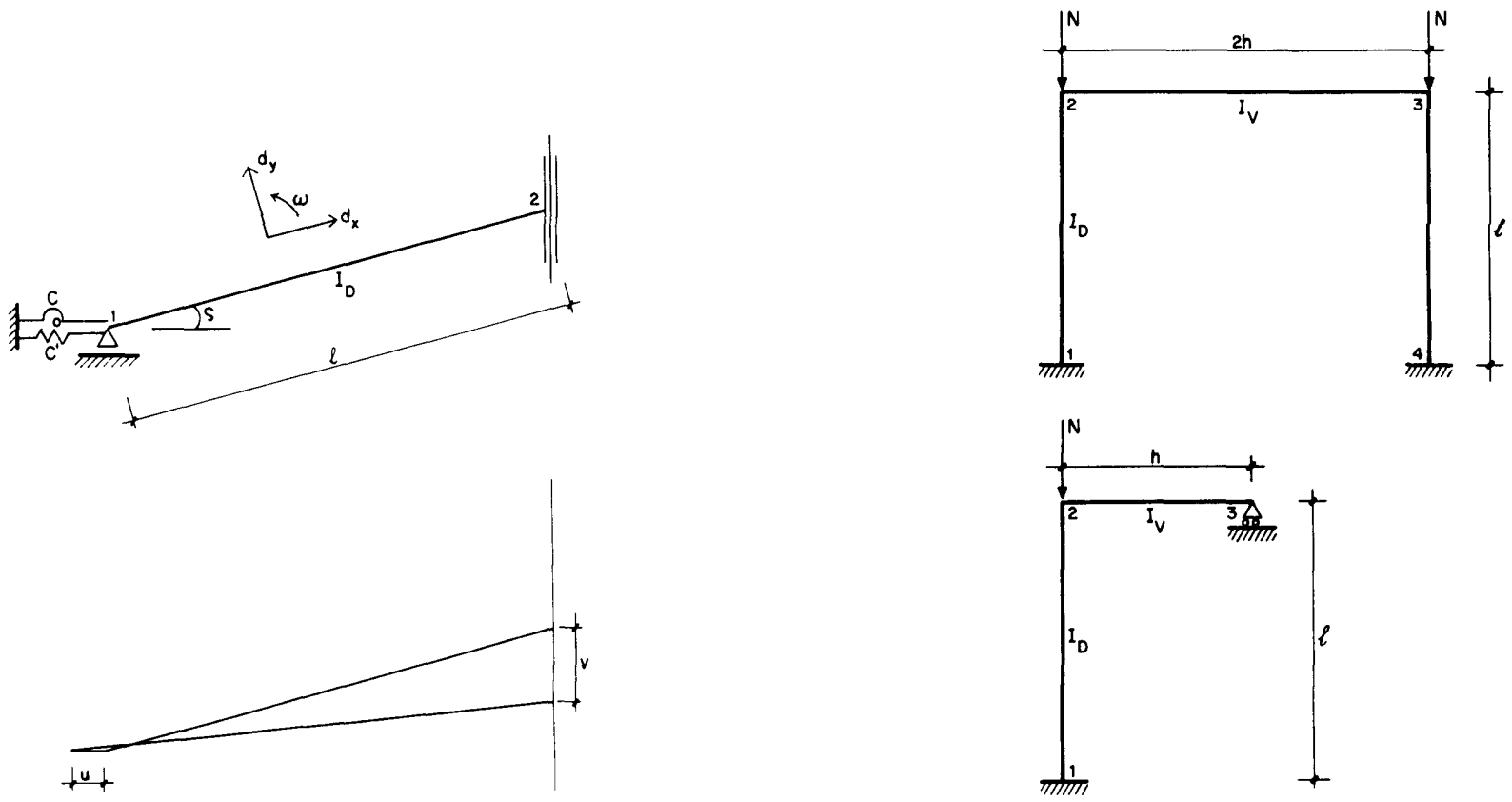

Fig. 4. Análisis del dintel.

Fig. 5. Pórtico equivalente para el análisis del dintel. 
Introduciendo las "funciones de estabilidad» de la barra (refs. 14 y 15), la matriz de rigidez de la misma, prescindiendo de las elongaciones de la directriz, se expresa:

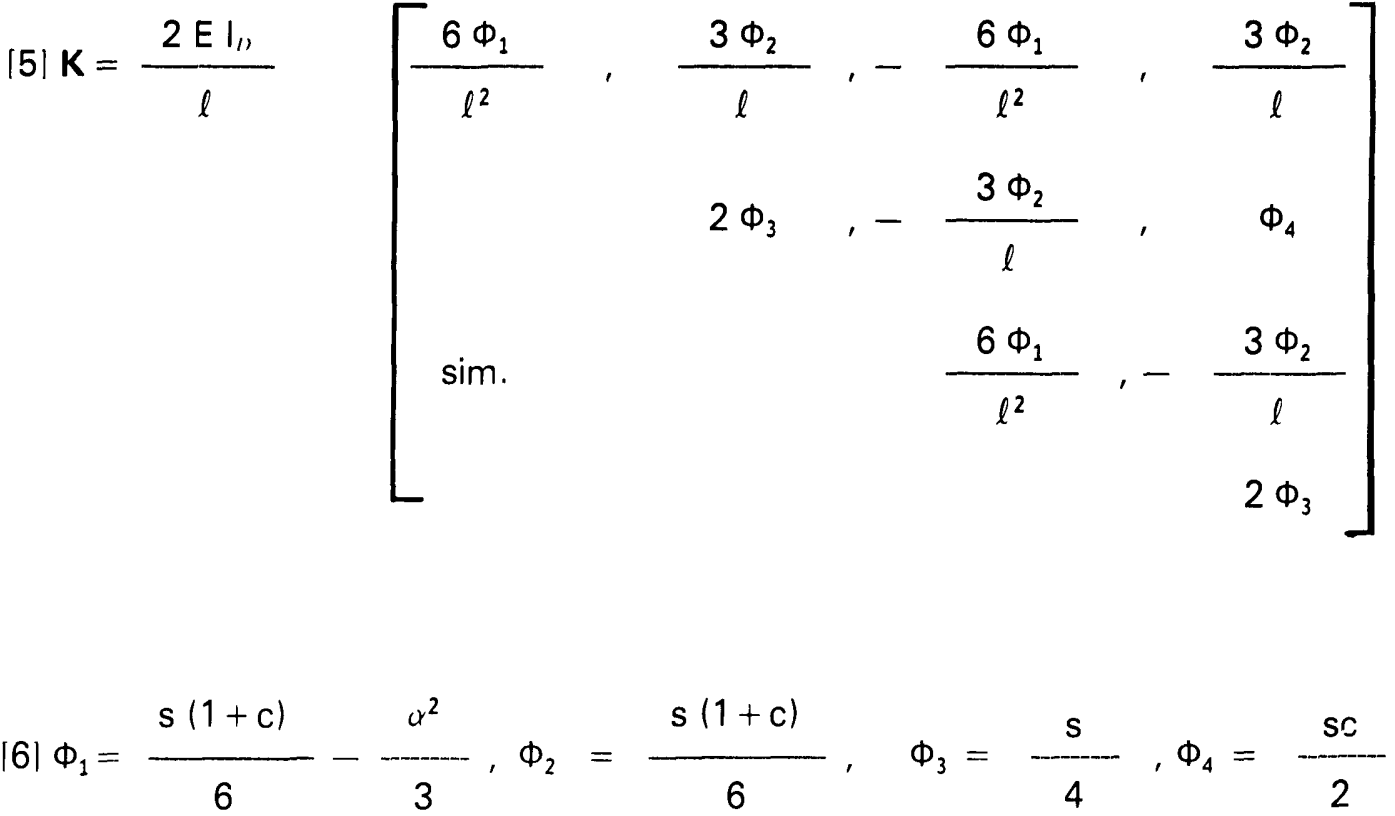

$$
\begin{aligned}
& \left\lceil 7 \mid \mathrm{s}=\frac{(1-2 \alpha \operatorname{ctg} 2 \alpha) \alpha}{\operatorname{tg} \alpha-\alpha} \quad ; \quad \mathrm{c}=\frac{2 \alpha-\operatorname{sen} 2 \alpha}{\operatorname{sen} 2 \alpha-2 \alpha \cos 2 \alpha}\right. \\
& {[8] \alpha=\frac{\pi}{2} \sqrt{\frac{\mathrm{N}^{*}}{\mathrm{~N}_{E}}} ; \quad \mathrm{N}_{E}=\frac{\left.\pi^{2} \mathrm{E}\right|_{t}}{\ell^{2}}} \\
& \text { Considerando la expresión de la energía elástica } U=\frac{1}{2} \mathbf{d}^{\prime} \mathrm{K} \mathbf{d}+\frac{1}{2} \mathrm{C} \omega_{1}^{2} \text { y tenien- }
\end{aligned}
$$$$
\text { do en cuenta }|4| \text {, se obtiene } U=\frac{1}{2} \mathbf{u}^{\prime} \mathbf{K}^{*} \mathbf{u} \text {, con: }
$$$$
|9| K^{*}=\frac{\left.2 E\right|_{1,}}{\ell}\left[\begin{array}{lll}
6 & \Phi_{1} / \operatorname{sen}^{4} \varrho, & 3 \Phi_{2} / \operatorname{sen}^{2} \varrho \\
3 & \Phi_{2} / \operatorname{sen}^{2} \varrho, 2 & \left(\Phi_{3}+\mu\right)
\end{array}\right]
$$

Anulando el determinante de la matriz de rigidez $\mathbf{K}^{*}$ se deduce la condición de inestabilidad:

$$
\text { [10] } 4 \Phi_{1} \cdot\left(\Phi_{3}+\mu\right)-3 \Phi_{2}^{2}=0
$$

Esta ecuación, en la que no intervienen el ángulo $\int$, es la misma ecuación que determina la carga crítica del pórtico traslacional representado en la fig. 5 inferior (siendo $I_{1}$. $=\mathrm{Ch} / 3 \mathrm{E}$ ), tal como puede comprobarse según un proceso análogo al precedente. A su vez, dicha carga crítica coincide evidentemente con la correspondiente al pandeo antimétrico del pórtico representado en la parte superior de la misma figura 5, la cual puede determinarse aplicando los criterios del mismo artículo de la ref. 1 mencionado en el apartado anterior. Por lo tanto, la longitud de 
pandeo del semidintel del pórtico original, vale $\beta \cdot \ell$, obteniéndose $\beta$ de la tabla 3.4. de la ref. 1 , con los siguientes datos:

[11] $K_{1}=1$

[12] $\mathrm{K}_{2}=\frac{2 \mathrm{I}_{s} / 3 \mathrm{~h}}{\frac{2 \mathrm{I}_{s}}{3 \mathrm{~h}}+\frac{\mathrm{I}_{1}}{\ell}}$ para pórticos biempotrados

[13] $\mathrm{K}_{2}=\frac{\mathrm{I}_{s} / 2 \mathrm{~h}}{\frac{\mathrm{I}_{s}}{2 \mathrm{~h}}+\frac{\mathrm{I}_{11}}{\ell}}$ para pórticos biarticulados

La longitud de pandeo del semidintel en el plano del pórtico oscila pues entre $\ell$ y $2 \ell$, pudiendo adoptar valores considerables; de esta forma, aun siendo en general la flexión del dintel la solicitación predominante del mismo, puede resultar determinante la comprobación a pandeo según el art. 3.9.2. de la ref. 1.

Para la aplicación de las anteriores expresiones, debe adoptarse el valor «efectivo» de la rigidez del soporte que se establece en el siguiente apartado.

\section{grados de empotramiento «efectivos»}

La presencia de un esfuerzo axil de compresión, $\mathrm{N}_{D}^{*}$, en el semidintel del pórtico, reduce la coacción elástica al giro que dicha barra introduce en el extremo superior del soporte, en el modo de pandeo antimétrico (fig. 2). Análogamente, la coacción $\mathrm{C}$ en el modo simétrico (fig. 4) adopta un valor "efectivo» apreciablemente menor que $4 \mathrm{El}_{s} / \mathrm{h}\left({ }^{*}\right)$ si la compresión del soporte, $\mathrm{N}_{s}^{*}$, es considerable. La omisión de estos efectos puede originar errores notables incluso en casos normales; por ejemplo, para un pórtico biempotrado, con $\mathrm{I}_{D}=\mathrm{I}_{S}, \frac{\mathrm{h}}{\ell}=0^{\prime} 616, \varrho=22 \frac{1^{\circ}}{2}$ y sometido a una carga vertical uniformemente repartida sobre el dintel, el error en el valor de la carga crítica sería del orden de un $35 \%$ (la solución "exacta» del caso indicado puede encontrarse en la ref. 19).

Las rigideces «efectivas» se obtienen aplicando a las rigideces «lineales», $3 \mathrm{El}_{t} /$ / y $4 \mathrm{El}_{s} / \mathrm{h}$, respectivamente los factores correctores siguientes (ref. 14):

$[14] \psi_{l}=\frac{1}{3} \underline{S} \cdot\left(1-\underline{C^{2}}\right)$
$[15] \psi_{s}=\frac{\underline{S}}{4}$

(*) En el presente apartado la base del soporte se supone empotrada en la cimentación, si bien la formulación final (apartado siguiente) contempla las dos posibilidades de apoyo articulado o empotrado. 
En estas expresiones, las funciones de estabilidad (s y c) de cada barra se determinan de acuerdo con [7], adoptando:

$$
\begin{aligned}
& \text { [16] } \alpha_{D}=\frac{\pi}{2} \sqrt{\gamma_{11}} ; \gamma_{11}=\frac{\mathrm{N}_{1}^{*} \ell^{2}}{\pi^{2} \mathrm{El}_{l}}=\frac{\mathrm{N}_{\prime \prime}^{*}}{\mathrm{~A}_{l}} \cdot \frac{\left(\ell / \mathrm{i}_{,}\right)^{2}}{\pi^{2} \mathrm{E}} \\
& \text { [17] } \alpha_{s}=\frac{\pi}{2} \sqrt{\gamma,} ; \gamma_{s}=\frac{\mathrm{N}_{s}^{*} h^{2}}{\pi^{2} \mathrm{El}}=\frac{\mathrm{N}_{s}^{*}}{\mathrm{~A}} \cdot \frac{\left(\mathrm{h} / \mathrm{i}_{\mathrm{s}}\right)^{2}}{\pi^{2} \mathrm{E}}
\end{aligned}
$$

siendo A é i el área y el radio de giro (correspondiente a la flexión en el plano del pórtico) de las secciones transversales de las barras.

Los coeficientes [14] y [15] pueden tomarse de la Tabla n. ${ }^{\circ} 1$, o bien, aproximadamente, de las expresiones siguientes:

$$
\begin{aligned}
& {[18] \psi_{D} \simeq 1-0,55 \gamma_{D}-0,45 \gamma_{D}^{2}\left(0 \leqslant \gamma_{D} \leqslant 1\right)} \\
& {[19] \psi_{s} \simeq 1-0,38 \gamma, \quad(0 \leqslant \gamma \leqslant 1)}
\end{aligned}
$$

Estos factores deben aplicarse a las inercias del dintel y del soporte en el cálculo de los grados de empotramiento de las piezas; así, las inercias "eficaces» de las piezas en la expresión [2], son $\psi_{D} I_{D}$ é $I_{s}$, mientras que en [12] los valores a aplicar son $I_{D}$ y $\psi_{s} I_{s}$.

Para piezas dimensionadas con arreglo a la vigente normativa española pueden acotarse estos coeficientes de manera conservadora, suponiendo que el cociente $N^{*} / A$ adopta el máximo valor posible en compresión centrada para una esbeltez de valor $\ell / \mathrm{i}_{D}$ ó $\mathrm{h} / \mathrm{i}_{\mathrm{s}}$ (respectivamente para el cálculo de $\psi_{D}$ y $\psi$.). De esta forma las rigideces «efectivas» resultan infravaloradas, ya que los valores permitidos de los axiles $\mathrm{N}^{*}$ serán claramente menores a los antedichos, debido en primer lugar a la existencia de otros esfuerzos, y en segundo lugar a que las esbelteces de cálculo de las piezas son superiores $a \ell / i_{D}$ y $h / i_{s}$, según se ha expuesto en los apartados precedentes. No obstante, esta manera de proceder tiene la ventaja de permitir formular de modo directo los valores de $\psi$ a partir de las características de las piezas, independientemente de su solicitación:

$$
\begin{aligned}
& {[20]_{\gamma} \simeq \frac{\lambda^{2}{ }_{R}}{\left(0,5+0,65 \lambda_{k}^{2}\right)+\sqrt{\left(0,5+0,65 \lambda_{k}^{2}\right)^{2}-\lambda_{R}^{2}}} ; \psi, \text { según [18] ó [19] }} \\
& {\left[21 \lambda_{R}=\frac{\ell}{\pi i_{D}} \sqrt{\frac{\sigma_{u}}{\mathrm{E}}} \text { o } \frac{\mathrm{h}}{\pi \mathrm{i}_{\mathrm{s}}} \sqrt{\frac{\sigma_{u}}{\mathrm{E}}}\right.}
\end{aligned}
$$

Los valores así calculados se expresan en la Tabla $n .^{\circ} 2$. Para acero del tipo A42, si se cumple $\mathrm{i}_{n} \geqslant \ell / 100$ é $\mathrm{i}_{\text {, }} \geqslant \mathrm{h} / 20,0$, pueden adoptarse los valores conservadores $\psi_{D}=0,50$ y $\psi_{\mathrm{v}}=0,72$, los cuales han sido incorporados a las fórmulas operativas expresadas en el siguiente apartado. 
Tabla $\mathbf{n}^{\circ} 1$.

Coeficientes de reducción de rigideces

\begin{tabular}{cccc}
$r$ & & $\psi_{\nu}$ & $\psi$ \\
\cline { 1 - 1 } 0 & & 1 & \\
0,1 & & 0,932 & 0,967 \\
0,2 & & 0,860 & 0,932 \\
0,3 & & 0,783 & 0,897 \\
0,4 & & 0,701 & 0,861 \\
0,5 & & 0,611 & 0,824 \\
0,6 & & 0,514 & 0,785 \\
0,7 & & 0,407 & 0,745 \\
0,8 & & 0,287 & 0,704 \\
0,9 & 0,153 & 0,661 \\
1 & & 0 & 0,617
\end{tabular}

Tabla . $^{\circ} 2$.

Coeficientes de reducción de rigideces (valores conservadores)

\begin{tabular}{ccccc}
$\lambda_{K}$ & & $\psi_{D}$ & & $\psi_{s}$ \\
\cline { 1 - 1 } 0 & & 1 & & 1 \\
0,25 & & 0,965 & & 0,977 \\
0,50 & & 0,851 & & 0,913 \\
0,75 & & 0,677 & & 0,835 \\
1 & & 0,527 & & 0,779 \\
1,25 & & 0,443 & & 0,750 \\
1,50 & & 0,398 & & 0,735 \\
1,75 & & 0,373 & & 0,727 \\
2 & & 0,357 & & 0,722 \\
2,25 & & 0,347 & & 0,719 \\
2,50 & & 0,339 & & 0,717 \\
2,75 & & 0,334 & & 0,715 \\
3 & & 0,330 & & 0,714
\end{tabular}

\section{conclusiones}

Para la comprobación de la inestabilidad en su propio plano del pórtico representado en la fig. 1 , como longitudes de pandeo de las barras pueden adoptarse los siguientes valores conservadores, salvo un estudio más preciso:

a) Soportes $\left(A B\right.$ y $\left.A^{\prime} B^{\prime}\right): \ell_{h}=\beta h$, determinando $\beta$ en la Tabla 3.4. de la ref. 1, con los datos siguientes:

$\mathrm{K}_{1}=1$ en pórticos biempotrados; cero en pórticos biarticulados.

$$
\mathrm{K}_{2}=\frac{0,25 \mathrm{I}_{D} / \ell}{0,25 \mathrm{I}_{D} / \ell+\mathrm{I}_{\mathrm{N}} / \mathrm{h}}
$$

Este criterio es válido si el radio de giro del dintel verifica la condición $\mathrm{i}_{l} \geqslant \ell / 100$, para piezas de acero A42.

b) Semidinteles $\left(B C\right.$ y $\left.B^{\prime} C^{\prime}\right)$ : $\ell_{\kappa}=\beta \ell$, determinando $\beta$ en la misma tabla, con:

$$
\begin{aligned}
& \mathrm{K}_{1}=1 \\
& \mathrm{~K}_{2}=\left\{\begin{array}{cc}
\frac{0,48 \mathrm{I}_{S} / \mathrm{h}}{0,48 \underline{\mathrm{I}}_{S} / \mathrm{h}+\mathrm{I}_{D} / \ell} & \begin{array}{c}
\text { en pórticos biempotrados } \\
\left(\mathrm{i}_{s} \geqslant \mathrm{~h} / 200\right)
\end{array} \\
\frac{0,25 \mathrm{I}_{S} / \mathrm{h}}{0,25 \underline{\mathrm{I}_{S}} / \mathrm{h}+\mathrm{I}_{D} / \ell} & \begin{array}{c}
\text { en pórticos biarticulados } \\
\left(\mathrm{i}_{s} \geqslant \mathrm{~h} / 100\right)
\end{array}
\end{array}\right.
\end{aligned}
$$

Estas últimas expresiones son válidas si el radio de giro de la sección del soporte cumple las limitaciones indicadas, en caso de acero A42.

La comprobación final de cada pieza debe efectuarse con arreglo a las prescripciones del artículo 3.9 de la ref. 1. 
notación
$A_{n,} A_{1}=$ Areas de las secciones transversales del dintel y dei soporte.
c = Segunda función de estabilidad
C. $C^{\prime}=$ Rigideces de coacciones eísticas a giro y desplazamiento.
$\mathrm{d}=$ vector de movimientos
E $\quad$ = Módulo de elasticidad.
h $=$ Altura del soporte.
$i, i=\quad=$ Radios de giro del dintel y ae suporte (flexión en el plano del póricol.
$i_{i} i_{\text {. }}=\mathrm{M}$. de $\mathrm{i}$. del dintel y del soporte (idem)
$K_{,} K^{*}=$ Matrices de rigidez.
$\mathrm{K}_{1}, \mathrm{~K}_{2}=$ Grados de empotramiento.
$\ell=$ Longitud del semidiritel
$\ell_{K} \quad=$ Longitud de pandeo.
$\mathrm{N}=$ Esfuerzo axil de compresicin
$\mathrm{N}_{\nu}^{*}, \hat{N}_{*}^{*}=$ Axiles ponderados del dintei y dei scponte

$\Phi_{1}, \Phi_{2}, \Phi_{3}, \Phi_{4}=$ Funciones de estabilidad derivadas

\section{referencias}

1. Norma básica MV-103 sobre Câculo de las estucturas de acero laminaúo en la edificación. Ministerio de la Viviendá. 1972

2. Instrucción e.m. 62 para estructiras de acero, segunda edición. instituto Eduardo Torroja. 1969.

3. Recommendations for Steel Constructions. European Convention for Constructional Steelwork (C.E.C.M.). 1976.

4. DIN 4114.

5. Règles CM66 de Calcul des constructions en acier. I.T.B.T.P., C.T.A.C.M. Déc 1966.

6. S. Timoshenko: "Teoria de la estabilicad ciastica». Ediar. 1961.

7. D.O. Brush, B.O. Aimroth: «Bucking of Bars. Piates and Sheils». McGraw-Hill, 1975

8. O Belluzzi: "Ciencia de la Construcoion». vol IV. Aguilar 1967.

9. V. Zignoli: "Construcciones metáliçsis. Dossat, 1978.

10. La Construcción metálica («Stahibau». E.T.S. de 1.C.C.P. Madrid, 1963 y 1965.

11. Bresier et al.: «Diseño de estrucuras de acero» Limusa-Wiley, 1973.

12. F.S. Merritt et al.: "Structural Steel Designer's Handbook». McGraw-rtill, $\$ 972$

13. J.D. Renton; "Stability of space frames by computer anaiysis». ASCE - SO, Aug. 1962

14. M.R. Horne. W. Merchant: "The Stability of Frames». Pergamon Press. 1965

15. R.K. Livesley: "Métodos matriciales para cálculo de estructuras». Blume. 1970.

16. E.F. Masur et al: "Stability of Frames in the Presence of Primary Bending Moments". Trans. Am. Soc. Civil. Engrs., 127 (1), p. 736 (1962).

17. H.L. Su: "On Stability of two - Dimensional Structural Frameworks», Proc. Inst. Civil Engers., 16, p. 143 (June 1960).

18. S.I. Minn: "The Determination of the Crtical Loads of Plane Frames», Structural Eng. 39 (7), p. 221 (July 1961).

19. K.I. Majid: «Non-Linear Structuresn. Eutrerworths, 1972.

20. Manual on Stability of Steel Structues. European Convention for Cunstructional Steelwork. 1976.

\section{Tésumé}

NOTICE RELATIVE A LA DETERMI NATION DES "LONGUEURS DE "FLAMBEMENT" AUX PORTIQUUES METALLIOUES A DEUX VERSANTS Jesús Ortiz Herrera, Dr ingénieur des Ponts et Chaussées

Dans la construction de halls industriels face à la solution de ferme isostatiquemen appuyee sur des supports, on utilise de plu en plus le portique métallique à deux versants, de grande et de moyenne portée.

Pour l'étude de l'instabilité sur le plan du portique, on fait appel à la méthode des portique, on fait appel a la méthode des t: Longueurs de flambementn qui permet une mais gènéralement accepté pour son efficacité.

Bien qu'il existe des techniques d'analyse númérique qui permeltaient d'aborder avec precision le probieme de l'instabilité, cette precision le probieme de l'instabilité, cette
notice ne vise pas à apporter des innovations à la manière dont le probleme es abordé mais, en acceptant la validité approximative oje la methode actuelle de pres a donner des regles d'application immediate et compatibles avec les indications émanant des normes espagnoles.

\section{suminary}

NOTE RELATIVE TO THE DETERMI NATION OF "BENDING LENGTHS" IN DOUELE-SLOPING METAL PORTAL FAMES

Dr. Jesus Ortiz Herrara. Civil Engineer

in the construction of industrial sheds, in contras to the use of a rib isostatically braced on supports, there has been a con stanily more frequent use of the doubleslopirig metal portal frame of small and mediun spans.

For the sluoy of the lack of stability on the plane of the portal frame, recourse is had
to the "bending lengths" method which makes possible a piece by piece check -a debatable procedure, but generally accepted on account of its operational nature.

Although there are techniques of numerical analysis which made it possible to approach the problem of instability with precision, this Note does not intend to introduce inno vations in the treatment of the problem, but rather, accepting the approximate validity of the preseni method of calculation proposes to provide rules of immediate application compatible with what is set out in Spanish standards.

\section{zusammenfassung}

ANMERKUNG BEZUEGLICH DER BESTIMMUNG DER «KNICKLAEN. GEN" VON METALL-SATTELDAE CHERN

Dr. Jesús Ortiz Herrera, Ingenieui

Beim Bau von Industriehallen werden gegenüber der Lösung isostatischer, auf Trägern abgestütztee Gewölbeverschalungen imme mehr Metall-Satteidächer geringer und mittlerer Spannweite eingesetzt.

Zur Untersuchung der Unbeständigkeit der Bogenbauten wird immer mehr auf die Methode der "Knicklängen» zurückgegriffen, welche eine Prüfung Teil für Teil ermöglicht. Es handeit sich um ein betreitbares, aber wegen seirier Ausführbarkeit allgemein anerkaintes Verfahren.

Obgleich Verfahren für nummerische Uniersuchungen vorliegen, welche einen prezisen vorgang gegen aas Problem der Unbeständigkeit ermöglichen könnten, liegt die Absicht dieser Beschreibung nicht darin, Neuheiten in die Problembehandlung einzu annähernden Gültigkeit der derzeitigen Rechenmethode sofortige Anwendungsregeln festzulegen, die mit der spanischen Normung verträglicht sind. 